\title{
Non-Hermitian Topological Invariants in Real Space
}

\author{
Fei Song, ${ }^{1}$ Shunyu Yao, ${ }^{1}$ and Zhong Wang ${ }^{1, *}$ \\ ${ }^{1}$ Institute for Advanced Study, Tsinghua University, Beijing, 100084, China
}

\begin{abstract}
The topology of non-Hermitian systems is drastically shaped by the non-Hermitian skin effect, which leads to the generalized bulk-boundary correspondence and non-Bloch band theory. The essential part in formulations of bulk-boundary correspondence is a general and computable definition of topological invariants. In this paper, we introduce a construction of non-Hermitian topological invariants based directly on real-space wavefunctions, which provides a general and straightforward approach for determining non-Hermitian topology. As an illustration, we apply this formulation to several representative models of non-Hermitian systems, efficiently obtaining their topological invariants in the presence of non-Hermitian skin effect. Our formulation also provides a dual picture of the non-Bloch band theory based on the generalized Brillouin zone, offering a unique perspective of bulk-boundary correspondence.
\end{abstract}

Non-Hermitian Hamiltonians have widespread applications in physics. For example, when a quantummechanical system is open, meaning that its interaction with the environment is nonnegligible, its effective Hamiltonian is non-Hermitian [1 5]. The ubiquitous loss and engineered gain in classical wave phenomena 6 [9], the finite quasiparticle lifetimes $10-15$, and certain statisticalmechanical models [16], etc, are naturally described in terms of non-Hermitian Hamiltonians. Recently, growing efforts have been invested in uncovering novel topological phases in non-Hermitian systems. Among other observations, we mention that non-Hermiticity calls for revised bulk-boundary correspondence [17 32] and novel topological invariants 19 21, 23, 33 40], introduces new symmetries that enrich the topological classifications of bands 41 43, brings in non-Hermitian topological semimetals exhibiting exceptional band degeneracies without Hermitian counterparts 44 54], and induces many other interesting phenomena [55 87$]$.

Crucial to understanding the band topology is the nonHermitian skin effect (NHSE) [19, 22, 24], namely the exponential localization of continuous-spectrum eigenstates to boundaries. Its meanings and consequences are under active studies 20, 25, 28 30, 34, 48, 88 99. In particular, NHSE suggests the non-Bloch bulk-boundary correspondence 19, 22], and leads to the non-Bloch band theory based on the generalized Brillouin zone (GBZ) [19, 20, 23, 33, 34.

The calculation of GBZ can be quite challenging in higher dimensions, and the definition of GBZ is unclear in the presence of disorders. Here, we construct real-space topological invariants that can be conveniently applied to these general cases. For the usual Hermitian bands, the Brillouin zone and real space are precisely connected by the Fourier transformation, enabling a simple translation of Brillouin-zone topological invariants into realspace ones (e.g., Refs. [100, 101]). At first glance, such a favorable picture is not to be expected in non-Hermitian bands, as the eigenstates lose the extendedness of Bloch waves by the NHSE. Somewhat surprisingly, we find that non-Hermitian bulk topological invariants remains defin- able in real-space wavefunctions (energy eigenstates). It provides an efficient tool for non-Hermitian topology.

Bipolar NHSE and the Bloch point.-To illustrate a few unnoticed features of NHSE, we design a nonHermitian Su-Schrieffer-Heeger (SSH) model with a $t_{3} \pm$ $\gamma / 2$ term [Fig:1(a)], which slightly differs from previous non-Hermitian SSH models [19, 23, 33, 36, 38, 56]. The Bloch Hamiltonian reads

$$
\begin{aligned}
H(k) & =d_{x}(k) \sigma_{x}+d_{y}(k) \sigma_{y} ; \\
d_{x}(k) & =t_{1}+\left(t_{2}+t_{3}\right) \cos k+i \frac{\gamma}{2} \sin k, \\
d_{y}(k) & =\left(t_{2}-t_{3}\right) \sin k+i \frac{\gamma}{2} \cos k,
\end{aligned}
$$

where $\sigma_{x, y, z}$ 's are the Pauli matrices in the $A, B$ sublattice space. As $\sigma_{z}$ is absent, this Hamiltonian has the sublattice symmetry (also called the chiral symmetry) $\sigma_{z}^{-1} H(k) \sigma_{z}=-H(k)$. Accordingly, the real-space Hamiltonian $H$ satisfies $S^{-1} H S=-H$, where the entries of $S$ are $S_{x s, y s^{\prime}}=\delta_{x y}\left(\sigma_{z}\right)_{s s^{\prime}}$, with $x, y$ referring to the unit cell and $s, s^{\prime}=A, B$ referring to the sublattice. The energy eigenvalues come in pairs $(E,-E)$, therefore the topological edge modes with $E=0$ are protected.

The Bloch-Hamiltonian eigenvalues are $E_{ \pm}(k)=$ $\pm \sqrt{d_{x}^{2}+d_{y}^{2}}$, and the energy gap closes at $t_{1}= \pm\left(t_{2}+\right.$ $\left.t_{3}\right) \pm \gamma / 2$. Conventional bulk-boundary correspondence would imply that topological transition points are among them. For example, these points are $t_{1}= \pm 1.15, \pm 1.25$ when $t_{2}=1, t_{3}=0.2, \gamma=0.1$. However, for a long open-boundary chain (OBC), one finds only two critical points at $t_{1} \approx \pm 1.21$, and zero modes exist for $t_{1} \in[-1.21,1.21]$ [Fig:1(d)]. Similar breakdowns of conventional bulk-boundary correspondence are known in other models (e.g., Refs. $18,19,22]$ ), and the underlying mechanism is the NHSE[19, 24].

To visualize the NHSE, it is helpful to plot all the energy eigenstates as a function of eigenenergies [Fig,2(a)]. Apparently, almost all eigenstates are localized at the boundaries. Moreover, a notable feature arises that both the two ends of chain accommodate eigenstates. The eigenstates with $|E| \lesssim 0.8(\gtrsim 0.8)$ are localized at the 
right (left) end. This "bipolar NHSE" inevitably leads to Bloch-wave-like extended eigenstates at $|E|=E_{B} \approx 0.8$, interpolating the left-localized and right-localized eigenstates. Eigenstates being extended only at several discrete energies, as seen here, seems to be unique to non-Hermitian systems, and these discrete energies are dubbed the "Bloch points". These Bloch points may have potential applications, e.g., as extended laser modes (Notably, the extendedness of eigenstates at Bloch points is robust to perturbations, which is unlike previous proposals such as Ref.[102], where perturbations generally destroy the extendedness of modes). The Bloch points and the concomitant bipolar NHSE vividly defy the oversimplified picture that the eigenstates are localized towards the direction of dominant hoppings (i.e., localized at the left end when $\left.\left|t_{3}+\gamma / 2\right|>\left|t_{3}-\gamma / 2\right|\right)$.

The Bloch point $E_{B}$ can be calculated from the feature that it belongs to both the Bloch spectrum and OBC spectrum. Here, we notice that the OBC energies are real-valued for $t_{1}>0.9(\mathrm{Fig} \mathbb{1}(\mathrm{c}))$, whereas the $H(k)$ eigenvalues $E_{ \pm}(k)$ are generally complex-valued except at several isolated $k$ points. Therefore, the Bloch points have to been searched within these real-eigenvalue points, which determines $k= \pm \arccos \left(-t_{1} / 2 t_{2}\right) \equiv \pm k_{B}$, and the Bloch energies

$$
\pm E_{B}= \pm E\left(k_{B}\right)= \pm \sqrt{\left(t_{2}-t_{3}\right)^{2}-\frac{\gamma^{2}}{4}} .
$$

For the parameters of Fig.2, $E_{B} \approx 0.8$. It happens to be independent of $t_{1}$, as confirmed numerically [Fig 2(c)].

GBZ approach.-The Bloch points are visible in the GBZ in the non-Bloch band theory [19, 23], which we briefly outline below for comparison with the real-space approach to be introduced. The Bloch phase factor $e^{i k}$ is generalized to $\beta$ (typically, $|\beta| \neq 1$ ), whose legitimate values form a one-dimensional trajectory dubbed GBZ in the complex plane. Particularly, the eigenvalues $E(\beta)$ of the GBZ Hamiltonian $H(k \rightarrow-i \ln \beta)$, or $H\left(e^{i k} \rightarrow \beta\right)$, are the continuous energy spectrums of OBC. This can be compared to the Hermitian cases where the eigenvalues of Hermitian $H(k)$ with $e^{i k}$ in the standard Brillouin zone (namely the unit cirlce) provide the OBC continuous spectrum. The precise shape of GBZ can be found as follows. According to the energy eigenvalue function, which is $E_{ \pm}(\beta)= \pm\left.\sqrt{d_{x}^{2}+d_{y}^{2}}\right|_{k \rightarrow-i \ln \beta}$ in our model, a given $E$ corresponds to several $\beta$ roots denoted by $\beta_{j}(E)$ 's, then the equation $\left|\beta_{i}(E)\right|=\left|\beta_{j}(E)\right|$ determines the legitimate values of $E$ and $\beta_{j}$ 's 19, 23] (More precisely, when ordered as $\left|\beta_{1}(E)\right| \leq\left|\beta_{2}(E)\right| \leq \cdots \leq\left|\beta_{N}(E)\right|$, the relevant $i, j$ are the middle two [23]), and these $\beta_{j}$ 's form the GBZ. Following this approach, we obtain the GBZ shown in Fig 2(b) for the present model. The bipolar NHSE manifests in the fact that the GBZ and unit circle intersect, as the interior $(\beta \mid<1)$ and exterior $(\beta \mid>1)$ of unit circle corresponds to exponential localization at the left
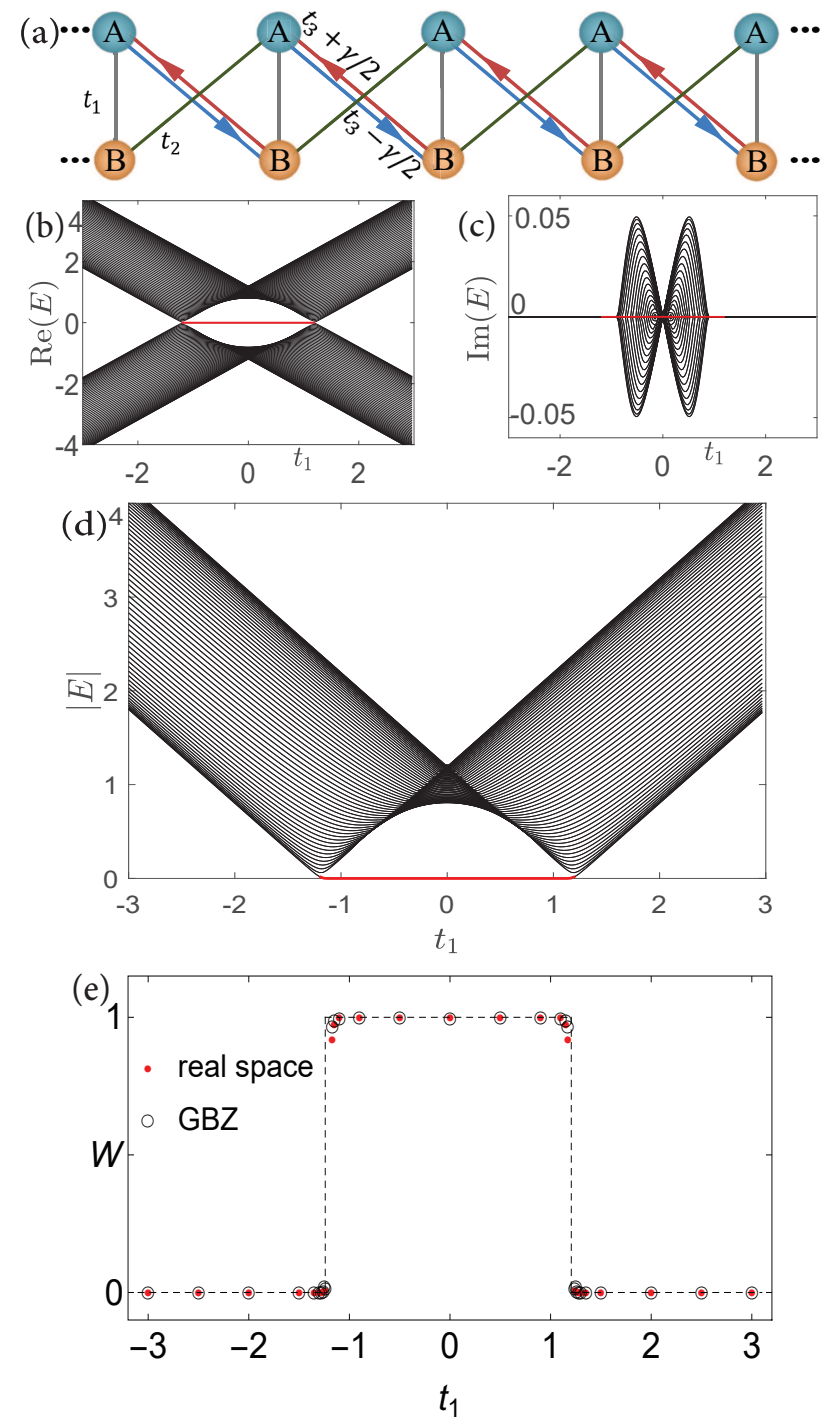

FIG. 1. (a) SSH model with alternating $t_{1}, t_{2}$ hoppings and a non-Hermitian $t_{3} \pm \gamma / 2$ hopping. (b)(c)(d) The OBC energy spectrum (real and imaginary part, and modulus) with length $L=80$ (in unit cell), with varying $t_{1}$ and fixed $t_{2}=1, t_{3}=$ $0.2, \gamma=0.1$. (e) Open-bulk winding number calculated in real space [red solid dots, by Eq.(5)] and non-Bloch winding number calculated in GBZ [black hollow dots, by Eq.(3)]. For the open-bulk winding number, the chain length $L=100$; the boundary cutoff $l=15$.

and right end, respectively. The Bloch points correspond exactly to the intersections.

Now we recall the construction of non-Bloch topological invariants from GBZ 19,23$]$. We start from the right and left eigenvectors of $H(k \rightarrow-i \ln \beta)$ (abbreviated as $H(\beta)$ below), defined via $H(\beta)\left|u_{R}(\beta)\right\rangle=E(\beta)\left|u_{R}(\beta)\right\rangle$ and $H^{\dagger}(\beta)\left|u_{L}(\beta)\right\rangle=E^{*}(\beta)\left|u_{L}(\beta)\right\rangle$, respectively. The chiral-symmetric partner has energy $-E(\beta)$ and $-E^{*}(\beta)$, and the corresponding right and left eigenvectors are referred to as $\left|\tilde{u}_{R}(\beta)\right\rangle$ and $\left|\tilde{u}_{L}(\beta)\right\rangle$. The right and left eigenvectors are chosen to satisfy the orthonormality [19]. The 

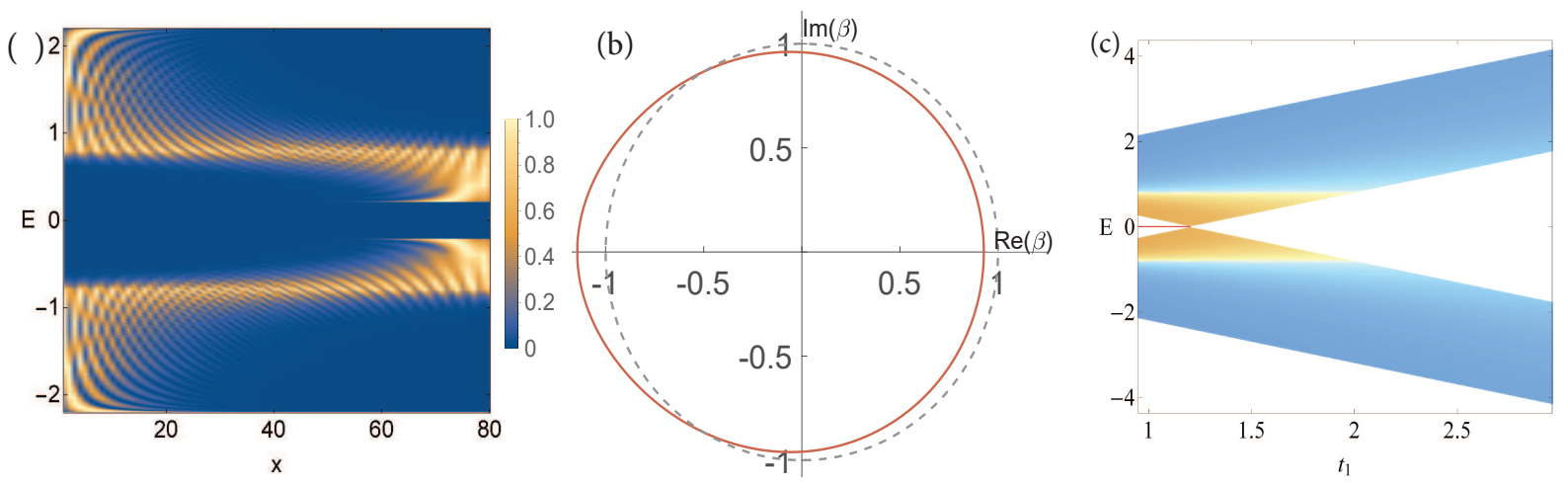

FIG. 2. (a) Profiles (modulus square) of all bulk energy eigenstates plotted as a function of eigenenergies; $x$ refers to the coordinate of open chain; $E$ refers to the eigenenergy. Eigenstate profiles are normalized to have maximum value 1 . Extended eigenstates exist only at the discrete "Bloch points" $E \approx \pm 0.8$; all other eigenstates are localized by NHSE. $t_{1}=t_{2}=1, t_{3}=$ $0.2, \gamma=0.1$. (b) shows the GBZ of (a). The dashed line is the unit circle. (c) Energy spectrum for varied $t_{1}$; other parameters the same as (a)(b). Yellow and blue color indicates eigenstate localization at the right and left end, respectively.

$Q$ matrix is then defined as $Q(\beta)=\left|u_{R}(\beta)\right\rangle\left\langle u_{L}(\beta)\right|-$ $\left|\tilde{u}_{R}(\beta)\right\rangle\left\langle\tilde{u}_{L}(\beta)\right|$. Finally, the non-Bloch winding number reads 19$]$

$$
W=\frac{1}{4 \pi i} \int_{\mathrm{GBZ}} \operatorname{Tr}\left[\sigma_{z} Q(\beta) d Q(\beta)\right]
$$

where the key ingredient is the integral path, namely the GBZ. This non-Bloch topological invariant determines the number of OBC zero modes [19], though their spatial profiles are not directly seen in GBZ.

Open-bulk topological invariant.-Motivated by the duality between the GBZ and real space, as exemplified in Fig2(a) and (b), we now construct topological invariants directly in real space. We begin with the OBC energy eigenstates. The right eigenstates satisfy $H|n R\rangle=E_{n}|n R\rangle$ and $H|\tilde{n} R\rangle=-E_{n}|\tilde{n} R\rangle$ (They are chiral-symmetry partners; $|\tilde{n} R\rangle$ is proportional to $S|n R\rangle$ ), and the left eigenstates satisfy $H^{\dagger}|n L\rangle=E_{n}^{*}|n L\rangle$ and $H^{\dagger}|\tilde{n} L\rangle=-E_{n}^{*}|\tilde{n} L\rangle$. They are orthonormal: $\langle m L \mid n R\rangle=\langle\tilde{m} L \mid \tilde{n} R\rangle=\delta_{m n},\langle m L \mid \tilde{n} R\rangle=\langle\tilde{m} L \mid n R\rangle=0$, which is satisfied when we write $H=T \Lambda T^{-1}$ with $\Lambda$ diagonal, and take the columns of $T$ and $\left(T^{-1}\right)^{\dagger}$ as the right and left eigenstates, respectively. We then introduce the open-boundary $Q$ matrix as

$$
Q=\sum_{n}(|n R\rangle\langle n L|-| \tilde{n} R\rangle\langle\tilde{n} L|),
$$

where $\sum_{n}$ is the sum over the eigenstates in the bulk continuous spectrum, leaving out the discrete edge modes.

With these preparations, the OBC bulk-band winding number (abbreviated as "open-bulk winding number") is defined as

$$
W^{\prime}=\frac{1}{2 L^{\prime}} \operatorname{Tr}^{\prime}(S Q[Q, X]),
$$

where $X$ is the coordinate operator, namely $X_{x s, y s^{\prime}}=$ $x \delta_{x y} \delta_{s s^{\prime}}$, and $\operatorname{Tr}^{\prime}$ stands for the trace over the middle interval with length $L^{\prime}$ (the total chain length is $L=L^{\prime}+2 l$, with the boundary intervals $1 \leq x \leq l$ and $l+L^{\prime}+1 \leq$ $x \leq L$ excluded from the trace). Ideally, $L \rightarrow \infty$ limit is assumed for Eq.(15), while in practice a modest size suffices. More explicitly, for the present model Eq.(5) reads $W^{\prime}=\frac{1}{2 L^{\prime}} \sum_{x=l+1}^{L-l} \sum_{s=A, B}(S Q[Q, X])_{x s, x s}$. Here, $l$ should be sufficiently large so that only the bulk information remains. In the Hermitian limit, this topological invariant reduces to Kitaev's formula [100, 101, 103 106], which can be intuitively understood by regarding $X$ as $i \partial_{k}$ for translationally invariant systems [100]. In view of the NHSE that destroys this intuition, it is a priori not obvious that the non-Hermitian generalization given by Eq.(5) is meaningful. It is even more worrisome to notice that, as a result of NHSE, $Q_{x s, y s^{\prime}}$ can grow exponentially with $y-x$ (This happens near the critical point $t_{1} \approx 1.21$ when other parameters take values as in Fig 1), though it is canceled out by the exponential decay of $Q_{y s^{\prime}, x s}$ in evaluating the trace in Eq.(5). Remarkably, these seemingly dangerous features do not invalidate the topological invariant, which is supported by our extensive numerical calculations.

Specifically, when applied to the model in Eq.(1), Eq.(5) accurately predicts the topological zero modes [Fig[1(e)]. Within numerical precision, it is found to be equal to Eq.(3), justifying our using the same symbol $W$.

Notably, in sharp contrast to the Hermitian cases for which the boundary condition is irrelevant, Eq.(5) corresponds to the topological edge modes only when $|n R\rangle,|n L\rangle$ are obtained from OBC. If instead periodicboundary condition is taken, the correspondence is generally lost in the presence of NHSE. To emphasize this unique non-Hermitian feature, the bulk winding number Eq.(5) is called an "open-bulk topological invariant".

Duality.-Now we show that Eq.(15) being equal to Eq.(3) is not accidental. While we do not have a mathematically strict proof of their being generally equal, we can give an "intuitive proof" with a few numerical inputs. 
First, we observe that the real-space $Q$ matrix has translational symmetry in the bulk, namely that when both $x$ and $y$ are far from the two ends of the chain, $Q_{x s, y s^{\prime}}$ depends only on the difference $x-y$ but not on $x, y$ separately, which allows us to write $Q_{x s, y s^{\prime}}=$ $Q_{s s^{\prime}}(x-y)$. This intuitive translational symmetry has been confirmed numerically. From the $Q(x)$ matrix we can construct a generalized "Fourier transformation":

$$
\tilde{Q}(\beta)=\sum_{x} Q(x) \beta^{-x} .
$$

It will now be helpful to establish a relation between this $\tilde{Q}(\beta)$ and the previous $Q(\beta)$ appearing in Eq.(3), which is constructed from the eigenstates of $H(\beta)$. Indeed, we have checked numerically (in this model and several other models) that the series $\tilde{Q}(\beta)$ is convergent in a domain of the complex $\beta$ plane, and that the GBZ can be smoothly deformed to a curve (denoted by "[GBZ]") in this domain without encountering any singularity (zero or divergence) of $Q(\beta)$. In fact, for most values of model parameters, we have found that GBZ itself belongs to the convergence domain and can be taken as [GBZ]. Intuitively, we expect the following relation

$$
\left.Q(\beta)\right|_{\beta \in[\mathrm{GBZ}]}=\left.\tilde{Q}(\beta)\right|_{\beta \in[\mathrm{GBZ}]},
$$

which has indeed been confirmed numerically. As such, we can write $\left.Q(\beta)\right|_{\beta \in[\mathrm{GBZ}]}=\sum_{x} Q(x) \beta^{-x}$. Inserting it into the non-Bloch winding number Eq.(3), we find that

$$
\begin{aligned}
W & =-\sum_{x, y} \int_{[\mathrm{GBZ}]} \frac{d \beta}{4 \pi i} \operatorname{Tr}\left[\sigma_{z} Q(x) \beta^{-x} y Q(y) \beta^{-y-1}\right] \\
& =\frac{1}{2} \sum_{x} \operatorname{Tr}\left[\sigma_{z} Q(x) x Q(-x)\right],
\end{aligned}
$$

where we have used $\int_{[\mathrm{GBZ}]} \beta^{-x-y-1} d \beta=2 \pi i \delta_{y,-x}$. Note that $x Q(-x)=[Q, X]_{y, y+x}$, which is independent of $y$ deep in the bulk. Therefore, we have

$$
W=W^{\prime},
$$

which identifies the non-Bloch topological invariant [Eq.(3)] and open-bulk topological invariant [Eq.(15)].

Open-bulk Chern number.-The construction of the open-bulk topological invariant is completely general. We now apply it to two-dimensional systems. The openbulk Chern number of a band $\alpha$ is given as

$$
C_{\alpha}=\frac{2 \pi i}{L_{x}^{\prime} L_{y}^{\prime}} \operatorname{Tr}^{\prime}\left(P_{\alpha}\left[\left[X, P_{\alpha}\right],\left[Y, P_{\alpha}\right]\right]\right),
$$

where $P_{\alpha}$ is the bulk-band projection operator,

$$
P_{\alpha}=\sum_{n \in \alpha}|n R\rangle\langle n L|,
$$

and other notations follow Eq.(5): The open-boundary system has a size $L_{x} \times L_{y}$, and $\operatorname{Tr}^{\prime}$ stands for the trace within the central area $L_{x}^{\prime} \times L_{y}^{\prime}\left(L_{i}^{\prime}=L_{i}-2 l_{i}\right.$, i.e.,

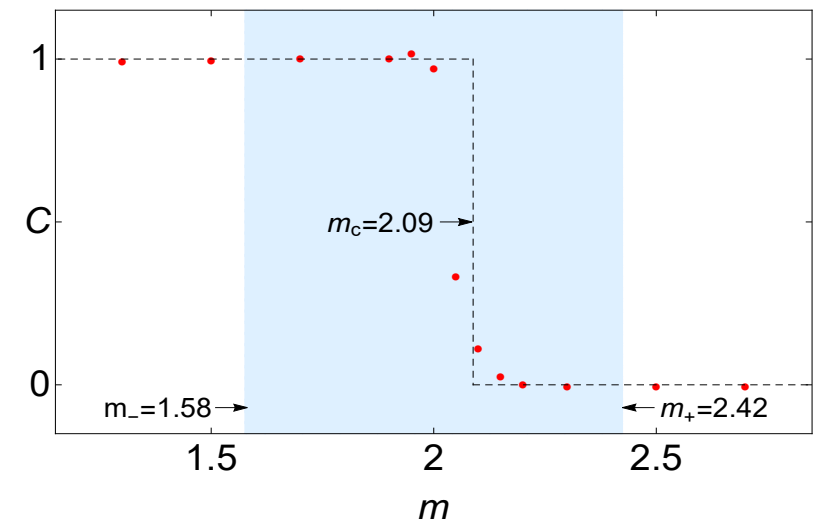

FIG. 3. Open-bulk Chern number $C$ for Eq.(12). Fixed parameters: $t=1, \gamma_{x}=\gamma_{y}=0.3, \gamma_{z}=0$. The size is $L_{x}=L_{y}=$ 30 and the truncation is $l_{x}=l_{y}=14$. The jump of $C$ occurs near the critical value $m_{c} \approx 2.09$ found in previous numerical energy spectrums 20]. The blue area $m \in\left[m_{-}, m_{+}\right]$stands for the gapless phase of the Bloch Hamiltonian $H\left(k_{x}, k_{y}\right)$, which is not seen in the open-boundary spectrums.

a boundary layer with thickness $l_{i}$ is removed). Note that the seemingly innocent $P_{\alpha}$ takes into account the non-Hermitian nature of the problem; for example, $P_{\alpha}$ is highly sensitive to the boundary condition in the presence of NHSE (taking periodic boundary condition would not work).

As an illustration, we calculate Eq.(10) for a simplest non-Hermitian Chern model [17, 20], whose Bloch Hamiltonian is

$$
\begin{aligned}
H\left(k_{x}, k_{y}\right)= & \left(\sin k_{x}+i \gamma_{x}\right) \sigma_{x}+\left(\sin k_{y}+i \gamma_{y}\right) \sigma_{y} \\
& +\left[m-t\left(\cos k_{x}+\cos k_{y}\right)+i \gamma_{z}\right] \sigma_{z} .
\end{aligned}
$$

This model has a pseudo-Hermitian symmetry which enables that most of the eigenenergies are real [20]. Because of the NHSE, the conventional bulk-boundary correspondence based on the topology of $H\left(k_{x}, k_{y}\right)$ breaks down. The open-bulk Chern number of the lower band is shown in Fig 3, which correctly tells the number of chiral edge modes found in previous numerical calculations 20], unambiguously establishing the non-Bloch bulk-boundary correspondence. Note that computing the non-Bloch Chern number in GBZ is much more challenging, and was done only in a continuum approximation [20].

Conclusions.-We have introduced non-Hermitian topological invariants defined in real space, which provides a general and efficient approach for understanding and computing non-Hermitian topology and bulkboundary correspondence. These open-bulk topological invariants can be conveniently evaluated with minimal input, and are generalizable to various non-Hermitian systems. Conceptually, this formulation is dual to the GBZ approach, which has the merit of being free of finite-size errors. The present approach has, however, the advantages of simplicity and convenience, and being applicable 
to disordered systems. As the two approaches have complementary virtues, their duality offers a versatile toolbox and deepened understandings for non-Hermitian topology.

Acknowledgements.-This work is supported by NSFC under Grant No. 11674189.

* wangzhongemail@gmail.com

[1] I. Rotter, Journal of Physics A: Mathematical and Theoretical 42, 153001 (2009).

[2] S. Diehl, E. Rico, M. A. Baranov, and P. Zoller, Nature Physics 7, 971 (2011).

[3] H. J. Carmichael, Phys. Rev. Lett. 70, 2273 (1993).

[4] F. Verstraete, M. M. Wolf, and J. I. Cirac, Nature physics 5, 633 (2009).

[5] S. Malzard, C. Poli, and H. Schomerus, Phys. Rev. Lett. 115, 200402 (2015).

[6] T. Ozawa, H. M. Price, A. Amo, N. Goldman, M. Hafezi, L. Lu, M. C. Rechtsman, D. Schuster, J. Simon, O. Zilberberg, and I. Carusotto, Rev. Mod. Phys. 91, 015006 (2019).

[7] L. Feng, R. El-Ganainy, and L. Ge, Nature Photonics 11, 752 (2017).

[8] R. El-Ganainy, K. G. Makris, M. Khajavikhan, Z. H. Musslimani, S. Rotter, and D. N. Christodoulides, Nature Physics 14, 11 (2018).

[9] B. Zhen, C. W. Hsu, Y. Igarashi, L. Lu, I. Kaminer, A. Pick, S.-L. Chua, J. D. Joannopoulos, and M. Soljačić, Nature 525, 354 (2015).

[10] H. Zhou, C. Peng, Y. Yoon, C. W. Hsu, K. A. Nelson, L. Fu, J. D. Joannopoulos, M. Soljačić, and B. Zhen, (2018), 10.1126/science.aap9859.

[11] H. Shen and L. Fu, Phys. Rev. Lett. 121, 026403 (2018).

[12] M. Papaj, H. Isobe, and L. Fu, Phys. Rev. B 99, 201107 (2019).

[13] V. Kozii and L. Fu, ArXiv e-prints (2017), arXiv:1708.05841 [cond-mat.mes-hall]

[14] T. Yoshida, R. Peters, and N. Kawakami, Phys. Rev. B 98, 035141 (2018)

[15] P. A. McClarty and J. G. Rau, arXiv e-prints , arXiv:1904.02160 (2019), arXiv:1904.02160 [cond-mat.str-el].

[16] N. Hatano and D. R. Nelson, Phys. Rev. Lett. 77, 570 (1996).

[17] H. Shen, B. Zhen, and L. Fu, Phys. Rev. Lett. 120, 146402 (2018).

[18] T. E. Lee, Phys. Rev. Lett. 116, 133903 (2016).

[19] S. Yao and Z. Wang, Phys. Rev. Lett. 121, 086803 (2018)

[20] S. Yao, F. Song, and Z. Wang, Phys. Rev. Lett. 121, 136802 (2018)

[21] D. Leykam, K. Y. Bliokh, C. Huang, Y. D. Chong, and F. Nori, Phys. Rev. Lett. 118, 040401 (2017).

[22] F. K. Kunst, E. Edvardsson, J. C. Budich, and E. J. Bergholtz, Phys. Rev. Lett. 121, 026808 (2018).

[23] K. Yokomizo and S. Murakami, Phys. Rev. Lett. 123, 066404 (2019).

[24] V. M. Martinez Alvarez, J. E. Barrios Vargas, M. Berdakin, and L. E. F. Foa Torres,
The European Physical Journal Special Topics 227, 1295 (2018)

[25] H.-G. Zirnstein, G. Refael, and B. Rosenow, arXiv e-prints , arXiv:1901.11241 (2019), arXiv:1901.11241 [cond-mat.mes-hall].

[26] Y. Xiong, Journal of Physics Communications 2, 035043 (2018).

[27] V. M. Martinez Alvarez, J. E. Barrios Vargas, and L. E. F. Foa Torres, Phys. Rev. B 97, 121401 (2018).

[28] C. H. Lee and R. Thomale, Phys. Rev. B 99, 201103 (2019)

[29] L. Jin and Z. Song, Phys. Rev. B 99, 081103 (2019).

[30] D. S. Borgnia, A. Jura Kruchkov, and R.-J. Slager, arXiv e-prints , arXiv:1902.07217 (2019), arXiv:1902.07217 [cond-mat.mes-hall],

[31] L. Herviou, J. H. Bardarson, and N. Regnault, Phys. Rev. A 99, 052118 (2019).

[32] S. R. Pocock, P. A. Huidobro, and V. Giannini, arXiv e-prints , arXiv:1902.00467 (2019), arXiv:1902.00467 [cond-mat.mes-hall]

[33] T.-S. Deng and W. Yi, Phys. Rev. B 100, 035102 (2019).

[34] T. Liu, Y.-R. Zhang, Q. Ai, Z. Gong, K. Kawabata, M. Ueda, and F. Nori, Phys. Rev. Lett. 122, 076801 (2019).

[35] Z. Gong, Y. Ashida, K. Kawabata, K. Takasan, S. Higashikawa, and M. Ueda, Phys. Rev. X 8, 031079 (2018)

[36] S. Lieu, Phys. Rev. B 97, 045106 (2018)

[37] K. Esaki, M. Sato, K. Hasebe, and M. Kohmoto, Phys. Rev. B 84, 205128 (2011)

[38] C. Yin, H. Jiang, L. Li, R. Lü, and S. Chen, Phys. Rev. A 97, 052115 (2018),

[39] H. Jiang, C. Yang, and S. Chen, Phys. Rev. A 98, 052116 (2018).

[40] A. Ghatak and T. Das, Journal of Physics: Condensed Matter 31, 263001 (2019).

[41] K. Kawabata, S. Higashikawa, Z. Gong, Y. Ashida, and M. Ueda, Nature Communications 10, 297 (2019).

[42] H. Zhou and J. Y. Lee, Phys. Rev. B 99, 235112 (2019).

[43] K. Kawabata, K. Shiozaki, M. Ueda, and M. Sato, arXiv e-prints , arXiv:1812.09133 (2018), arXiv:1812.09133 [cond-mat.mes-hall].

[44] Y. Xu, S.-T. Wang, and L.-M. Duan, Phys. Rev. Lett. 118, 045701 (2017).

[45] Z. Yang and J. Hu, Phys. Rev. B 99, 081102 (2019).

[46] A. Cerjan, M. Xiao, L. Yuan, and S. Fan, Phys. Rev. B 97, 075128 (2018).

[47] T. Yoshida, R. Peters, N. Kawakami, and Y. Hatsugai, Phys. Rev. B 99, 121101 (2019)

[48] H. Wang, J. Ruan, and H. Zhang, Phys. Rev. B 99, 075130 (2019)

[49] J. C. Budich, J. Carlström, F. K. Kunst, and E. J. Bergholtz, Phys. Rev. B 99, 041406 (2019)

[50] R. Okugawa and T. Yokoyama, Phys. Rev. B 99, 041202 (2019).

[51] K. Moors, A. A. Zyuzin, A. Y. Zyuzin, R. P. Tiwari, and T. L. Schmidt, Phys. Rev. B 99, 041116 (2019).

[52] J. Carlström and E. J. Bergholtz, Phys. Rev. A 98, 042114 (2018).

[53] H. Zhou, J. Y. Lee, S. Liu, and B. Zhen, Optica 6, 190 (2019).

[54] A. A. Zyuzin and A. Y. Zyuzin, Phys. Rev. B 97, 041203 (2018).

[55] G. Harari, M. A. Bandres, Y. Lumer, M. C. Rechtsman, 
Y. D. Chong, M. Khajavikhan, D. N. Christodoulides, and M. Segev, Science 359, eaar4003 (2018).

[56] B. Zhu, R. Lü, and S. Chen, Phys. Rev. A 89, 062102 (2014).

[57] S. Lieu, Phys. Rev. B 98, 115135 (2018).

[58] C. Yuce, Phys. Rev. A 93, 062130 (2016)

[59] C. Yuce, Physics Letters A 379, 1213 (2015).

[60] H. Menke and M. M. Hirschmann, Phys. Rev. B 95, 174506 (2017).

[61] T. M. Philip, M. R. Hirsbrunner, and M. J. Gilbert, Phys. Rev. B 98, 155430 (2018)

[62] Y. Chen and H. Zhai, Physical Review B 98, 245130 (2018).

[63] J. A. S. Lourenço, R. L. Eneias, and R. G. Pereira, Phys. Rev. B 98, 085126 (2018)

[64] M. Klett, H. Cartarius, D. Dast, J. Main, and G. Wunner, Phys. Rev. A 95, 053626 (2017)

[65] K. Kawabata, Y. Ashida, H. Katsura, and M. Ueda, Phys. Rev. B 98, 085116 (2018)

[66] J. M. Zeuner, M. C. Rechtsman, Y. Plotnik, Y. Lumer, S. Nolte, M. S. Rudner, M. Segev, and A. Szameit, Phys. Rev. Lett. 115, 040402 (2015)

[67] L. Xiao, X. Zhan, Z. H. Bian, K. K. Wang, X. Zhang, X. P. Wang, J. Li, K. Mochizuki, D. Kim, N. Kawakami, W. Yi, H. Obuse, B. C. Sanders, and P. Xue, Nature Physics 13, 1117 (2017).

[68] C. Wang and X. Wang, arXiv preprint arXiv:1901.06982 (2019).

[69] M. R. Hirsbrunner, T. M. Philip, and M. J. Gilbert, Phys. Rev. B 100, 081104 (2019).

[70] K. Kawabata, T. Bessho, and M. Sato, Phys. Rev. Lett. 123, 066405 (2019)

[71] C. Poli, M. Bellec, U. Kuhl, F. Mortessagne, and H. Schomerus, Nature communications 6, 6710 (2015).

[72] S. Weimann, M. Kremer, Y. Plotnik, Y. Lumer, S. Nolte, K. Makris, M. Segev, M. Rechtsman, and A. Szameit, Nature materials 16, 433 (2017).

[73] X. Zhan, L. Xiao, Z. Bian, K. Wang, X. Qiu, B. C. Sanders, W. Yi, and P. Xue, Phys. Rev. Lett. 119, 130501 (2017)

[74] M. S. Rudner and L. S. Levitov, Phys. Rev. Lett. 102, 065703 (2009).

[75] A. Cerjan, S. Huang, M. Wang, K. P. Chen, Y. Chong, and M. C. Rechtsman, Nature Photonics , 1 (2019).

[76] A. McDonald, T. Pereg-Barnea, and A. A. Clerk, Phys. Rev. X 8, 041031 (2018).

[77] Q.-B. Zeng, Y.-B. Yang, and Y. Xu, arXiv e-prints , arXiv:1901.08060 (2019), arXiv:1901.08060 [cond-mat.mes-hall]

[78] L. Zhou and J. Gong, Phys. Rev. B 98, 205417 (2018)

[79] W. Hu, H. Wang, P. P. Shum, and Y. D. Chong,
Phys. Rev. B 95, 184306 (2017)

[80] P. San-Jose, J. Cayao, E. Prada, and R. Aguado, Scientific reports 6, 21427 (2016).

[81] J. Avila, F. Peñaranda, E. Prada, P. San-Jose, and R. Aguado, arXiv e-prints , arXiv:1807.04677 (2018), arXiv:1807.04677 [cond-mat.mes-hall].

[82] W. B. Rui, Y. X. Zhao, and A. P. Schnyder, Phys. Rev. B 99, 241110 (2019).

[83] K. Y. Bliokh and F. Nori, Phys. Rev. Lett. 123, 054301 (2019)

[84] X.-W. Luo and C. Zhang, Phys. Rev. Lett. 123, 073601 (2019).

[85] M. G. Silveirinha, Phys. Rev. B 99, 125155 (2019)

[86] K. Yamamoto, M. Nakagawa, K. Adachi, K. Takasan, M. Ueda, and N. Kawakami, Phys. Rev. Lett. 123, 123601 (2019).

[87] Z. Ozcakmakli Turker and C. Yuce, Phys. Rev. A 99, 022127 (2019)

[88] H. Jiang, L.-J. Lang, C. Yang, S.-L. Zhu, and S. Chen, Phys. Rev. B 100, 054301 (2019)

[89] C. H. Lee, L. Li, and J. Gong, Phys. Rev. Lett. 123, 016805 (2019)

[90] F. K. Kunst and V. Dwivedi, Phys. Rev. B 99, 245116 (2019)

[91] F. Song, S. Yao, and Z. Wang, Phys. Rev. Lett. 123, 170401 (2019).

[92] E. Edvardsson, F. K. Kunst, and E. J. Bergholtz, Phys. Rev. B 99, 081302 (2019).

[93] M. Ezawa, Phys. Rev. B 100, 045407 (2019).

[94] X. Yang, Y. Cao, and Y. Zhai, arXiv preprint arXiv:1904.02492 (2019).

[95] M. Ezawa, Phys. Rev. B 99, 201411 (2019)

$[96]$ B. X. Wang and C. Y. Zhao, Phys. Rev. B 98, 165435 (2018).

[97] N. Okuma and M. Sato, Phys. Rev. Lett. 123, 097701 (2019)

[98] M. Ezawa, Phys. Rev. B 99, 121411 (2019)

[99] Z.-Y. Ge, Y.-R. Zhang, T. Liu, S.-W. Li, H. Fan, and F. Nori, Phys. Rev. B 100, 054105 (2019).

[100] A. Kitaev, Annals of Physics 321, 2 (2006).

[101] R. Bianco and R. Resta, Phys. Rev. B 84, 241106 (2011).

[102] S. Longhi, Annalen der Physik 530, 1800023 (2018).

[103] E. Prodan, New Journal of Physics 12, 065003 (2010).

[104] E. Prodan, T. L. Hughes, and B. A. Bernevig, Phys. Rev. Lett. 105, 115501 (2010).

[105] Y. X. Zhao, Y. Lu, and H.-Z. Lu, arXiv e-prints , arXiv:1706.09783 (2017), arXiv:1706.09783 [cond-mat.mes-hall].

[106] M. D. Caio, G. Möller, N. R. Cooper, and M. Bhaseen, Nature Physics 15, 257 (2019). 